\title{
Valorização do bagaço de cana-de-açúcar na produção de painéis aglomerados de baixa densidade
}

\author{
Suzane Sarno Soares ${ }^{1}$, José Benedito Guimarães Júnior ${ }^{2 *}$, Lourival Marin Mendes ${ }^{3}$, Rafael Farinass Men- \\ des $^{2}$, Thiago de Paula Protásio ${ }^{3}$, Fernando Jesus Noraga Lisboa ${ }^{4}$ \\ ${ }^{1}$ Curso de Engenharia Florestal, Universidade Federal de Goiás, Regional Jataí, Jataí - Brasil. \\ ${ }^{2}$ Departamento de Engenharia, Universidade Federal de Lavras, Lavras - Brasil. \\ ${ }^{3}$ Departamento de Ciências Florestais, Universidade Federal de Lavras, Lavras - Brasil. \\ ${ }^{4}$ Departamento de Ciências Florestais, Universidade Estadual Paulista, Botucatu - Brasil.
}

\begin{abstract}
RESUMO Há necessidade de diversificação do setor de painéis reconstituídos por meio da introdução de resíduos lignocelulósicos no processo produtivo. Além de agregar valor a materiais residuais, isso poderá contribuir para o aumento da produção industrial de painéis sem a necessidade de implantação de novos plantios florestais. O objetivo desse trabalho foi avaliar as propriedades físicas e mecânicas de painéis aglomerados de baixa densidade produzidos com diferentes quantidades de bagaço de cana-de-açúcar e madeira de Eucalyptus grandis. Os painéis foram produzidos com o adesivo ureia-formaldeído na proporção de $10 \%$ em relação à massa do painel. Na produção dos painéis foi utilizada pré-prensagem a $0,5 \mathrm{MPa}$ durante 10 minutos seguida de 4,0 $\mathrm{MPa}$ e $160^{\circ} \mathrm{C}$ durante 15 minutos. As porcentagens de resíduo utilizadas foram: 0, 20, 40 e $60 \%$ em associação com madeira de eucalipto. Para a avaliação da qualidade das chapas foram realizados ensaios físicos e mecânicos. O experimento foi arranjado em delineamento inteiramente casualizado, com três repetições. Para avaliação dos dados foi utilizada análise regressão linear simples. Constatou-se que a absorção de água não foi afetada pela inserção de bagaço de cana no painel, enquanto para inchamento em espessura, o aumento da proporção deste resíduo resultou em acréscimo nesta propriedade. As propriedades mecânicas foram afetadas negativamente com a inserção do bagaço de cana-de-açúcar. Os painéis produzidos com o bagaço de cana-de-açúcar e madeira não se adequaram à norma CS 236-66 no que se refere ao módulo de elasticidade na flexão estática. Os resultados demonstraram que até $26 \%$ de bagaço de cana-de-açúcar poderia ser utilizado em associação com a madeira de eucalipto na produção de painéis aglomerados de baixa densidade.
\end{abstract}

Palavras-chave: propriedades físico-mecânicas, material lignocelulósico, aproveitamento de resíduo.

\section{Valorization of sugarcane bagasse for production of low density particleboards}

\begin{abstract}
It's necessary to diversify the reconstituted panels sector by introducing lignocellulosic wastes in the production process. In addition to adding value to wastes materials, this may contribute to the increase the industrial production of panels eliminating the need of new forest plantations. The aim of this study was to evaluate the physical and the mechanical properties of low density particleboards produced with different amounts of sugarcane bagasse and Eucalyptus grandis wood. The panels were produced with $10 \%$ of urea formaldehyde adhesive (based on dry weight of particles). The pressing cycle consisted by: pre-pressing of $0.5 \mathrm{MPa}$ during 10 minutes followed by pressing of $4.0 \mathrm{MPa}$ and temperature at $160^{\circ} \mathrm{C}$ for 15 minutes. The following percentages of wastes were used: $0,20,40$ and $60 \%$ in association to eucalypt wood. For the evaluation of the quality of the panels, physical and mechanical tests were performed. The experiment was carried out according a completely randomized design with three replications. For data analysis, simple linear regression was used. It was verified that the water absorption was not affected by the insertion of sugarcane bagasse in the panels, while for thickness swelling, the increase of the proportion of the waste resulted in an increase of this property. The mechanical properties were negatively affected by the inclusion of sugarcane bagasse. The panels produced with sugarcane bagasse and wood did not reach the standard CS 236-66 for modulus of elasticity at static bending. The results showed that $26 \%$ of sugarcane bagasse could be added for production of particleboards associated to eucalypt wood
\end{abstract}

Keywords: physical-mechanical properties, lignocellulosic material, waste utilization. 


\section{Introdução}

O setor florestal brasileiro desempenha relevante importância para o produto interno bruto nacional por meio da geração de emprego e renda, sendo que as tendências e necessidades da atualidade demonstram um aumento da participação das florestas plantadas, no desenvolvimento socioeconômico de várias regiões do Brasil.

A cadeia produtiva florestal associada aos plantios de $E$ ucalyptus e Pinus caracteriza-se pela grande diversidade de produtos, podendo-se mencionar os painéis de madeira reconstituída. No entanto, há necessidade de diversificação desse setor por meio da introdução de novas matérias-primas no processo produtivo. Além de agregar valor a materiais residuais, isso poderá contribuir para o aumento da produção industrial de painéis sem a necessidade de implantação de novos plantios florestais (PROTÁSIO et al., 2015).

Dessa forma, tem sido reportado na literatura que os diferentes materiais lignocelulósicos, como os resíduos florestais e agroindustriais, podem ser utilizados na produção de painéis aglomerados (FIORELLI et al., 2011; SCATOLINO et al., 2017). Outra grande vantagem das chapas aglomeradas é a possibilidade de diminuir a variabilidade da madeira ou da biomassa residual que é oriunda de fatores químicos, físicos, mecânicos e anatômicos intrínsecos (PROTÁSIO et al., 2015).

Associado a isso, o aproveitamento dos resíduos lignocelulósicos pode ser uma alternativa para agregação de valor, contribuindo, ainda, com a minimização dos impactos ambientais, sociais e econômicos envolvidos na geração dos materiais residuais (RIBEIRO; MORELLI, 2009).

Nesse sentido, diferentes autores têm divulgado trabalhos relacionados à utilização de resíduos agrícolas, agroindustriais e florestais na produção de painéis aglomerados, como o bagaço de cana-de-açúcar (BATTISTELLE et al., 2009; FIORELLI et al., 2011; FREIRE et al., 2011; MENDES et al.,
2012); sabugo de milho (SCATOLINO et al., 2013, 2015); casca de mamona (SILVA et al., 2016); casca de arroz (CÉSAR et al., 2017); bagaço de sorgo (GUIMARÃES JUNIOR et al., 2016); pergaminho do grão de café (SCATOLINO et al., 2017); madeira do cafeeiro (CÉSAR et al., 2014); podas de erva mate (CARVALHO et al., 2015); pseudocaule da bananeira (GUIMARÃES et al., 2014) e palha de milho (SILVA et al., 2015).

Conforme supracitado, os resíduos de origem agroindustrial apresentam possibilidade de serem aproveitados na produção de painéis aglomerados (FIORELLI et al., 2011), com destaque para o bagaço de cana-de-açúcar (FREIRE et al., 2011); haja vista que este material apresenta características químicas similares a madeira e produção em larga escala no Brasil (DIAS et al., 2012; COMPANHIA NACIONAL DE ABASTECIMENTO, 2017).

Entretanto, um dos maiores entraves tecnológicos observados quanto à utilização do bagaço de cana-de-açúcar, como matéria-prima no setor de painéis reconstituídos, está na instabilidade dimensional das chapas comparativamente aos painéis confeccionados exclusivamente com partículas de madeira (FREIRE et al., 2011).

Dessa forma, uma das alternativas para mitigação de tal problemática, sugerida por Guimarães Junior et al. (2016), é o estudo da substituição de parte da madeira por materiais lignocelulósicos. Esses autores mencionaram que se faz necessário conhecer a quantidade de resíduo, em associação com a madeira, que possibilite adequação das propriedades física e mecânicas das chapas às normas de comercialização (CS 236-66, 1968).

Diante do exposto, o objetivo deste trabalho foi avaliar a qualidade física e mecânica de painéis aglomerados de baixa densidade produzidos com diferentes proporções de madeira (Eucalyptus grandis) e bagaço de cana-de-açúcar. 


\section{Material e Métodos}

O bagaço de cana-de-açúcar utilizado foi proveniente de uma indústria de produção de álcool, localizada em Jataí-GO. A madeira de Eucalyptus grandis foi proveniente de plantio experimental instalado em Jataí, Goiás, na Universidade Federal de Goiás (1753’S e 5243’ W), com 72 meses de idade.

A determinação da densidade básica da madeira foi realizada pelo método de imersão em água e seguindo as diretrizes da NBR 11941 (ABNT, 2003), sendo realizadas quatro repetições. A densidade básica do bagaço de cana-de-açúcar foi determinada pelo método da proveta ou método do deslocamento de água, considerando-se quatro repetições. As amostras, com aproximadamente $10 \mathrm{~g}$, previamente saturadas, foram imersas em uma proveta graduada com capacidade de $250 \mathrm{~mL}$, contendo $200 \mathrm{~mL}$ de água. Após a imersão, registrouse o volume de água deslocada, que corresponde ao volume da amostra. Posteriormente, levaram-se as amostras à estufa de circulação forçada a $103 \pm 2{ }^{\circ} \mathrm{C}$, para a obtenção da massa seca (PROTÁSIO et al., 2013a).

Para avaliação química, os materiais foram transformados em serragem em moinho tipo Willey. Posteriormente, as partículas foram classificadas em peneiras de 40 e 60 mesh e climatizadas em ambiente com $65 \pm 3 \%$ de umidade relativa e temperatura de $20 \pm 2^{\circ} \mathrm{C}$. A caracterização química dos materiais lignocelulósicos foi realizada considerando quatro repetições.

Os teores de extrativos solúveis em acetona e água quente (extrativos totais), lignina insolúvel em ácido sulfúrico e cinzas foram determinados segundo as normas NBR 14853 (ABNT, 2010a), NBR 7989 (ABNT, 2010b) e NBR 13999 (ABNT, 2003), respectivamente.

Os materiais lignocelulósicos foram processados em moinho de facas para a obtenção das partículas. Estas foram peneiradas, sendo utilizadas para a produção dos painéis aque- las que ficaram retidas entre as peneiras 20 e 40 mesh. Posteriormente, as partículas foram secas em estufa com circulação de ar forçada até a umidade na base seca de 3\%.

Os painéis foram produzidos com $0,20 \%, 40 \%$ e $60 \%$ de bagaço de cana-de-açúcar e, consequentemente, $100 \%, 80 \%$, $60 \%$ e $40 \%$ de madeira de eucalipto. As doze chapas aglomeradas produzidas foram calculadas com densidade nominal de $0,60 \mathrm{~g} / \mathrm{cm}^{3}$. O adesivo ureia-formaldeído, na proporção de 10\% (base teor de sólidos resinosos de 63,5\%), foi utilizado para o encolamento das partículas. As demais características do adesivo são: viscosidade de $479 \mathrm{cP}$ e pH de 8,94.

Na sequência, as partículas de bagaço de cana-de-açúcar e madeira de eucalipto foram misturadas manualmente com adesivo ureia-formaldeído e levadas a uma caixa formadora de colchão, com dimensões de $25 \mathrm{~cm}$ x $25 \mathrm{~cm}$ x $1,5 \mathrm{~cm}$. Não foi utilizada emulsão de parafina. O colchão de partículas foi então pré-prensado a $0,5 \mathrm{MPa}$, por $10 \mathrm{~min}$, à temperatura ambiente. Posteriormente, o painel passou pelo ciclo de prensagem, com $160^{\circ} \mathrm{C}$ e $4 \mathrm{MPa}$, por um período de $15 \mathrm{~min}$, obtendo-se os painéis homogêneos. Procedimento similar foi utilizado por Guimarães Junior et al. (2016).

Após finalização da fabricação dos painéis e climatização à temperatura de $25 \pm 2^{\circ} \mathrm{C}$ e a $65 \pm 3 \%$ de umidade relativa do ar, foram retirados os corpos de prova para determinação da qualidade físicas e mecânica das chapas.

Os módulos de elasticidade e ruptura no ensaio de flexão estática foram realizados de acordo com a norma DIN 52362 (1982). Já os testes de tração perpendicular, absorção de água após 2 e 24 horas de imersão em água, inchamento em espessura após 2 e 24 horas de imersão em água e densidade aparente foram baseados na norma D1037-100 (ASTM, 1980).

A razão de compactação foi obtida conforme equação 1 (GUIMARÃES JUNIOR et al., 2016; SCATOLINO et al., 2017):

$\mathrm{RC}=\frac{\mathrm{DP}}{\mathrm{DB}_{\mathrm{BC}} \mathrm{x}\left(\mathrm{BC}_{\%}\right)+\mathrm{DB}_{\mathrm{ME}} \mathrm{x}\left(\mathrm{ME}_{\%}\right)}$ 
Em que: RC é a razão de compactação; DP é a densidade aparente do painel aglomerado $\left(\mathrm{g} / \mathrm{cm}^{3}\right) ; \mathrm{DB}_{\mathrm{BC}}$ é a densidade básica do bagaço de cana-de-açúcar $\left(\mathrm{g} / \mathrm{cm}^{3}\right) ; \mathrm{BC}_{\%}$ é a porcentagem de bagaço de cana-de-açúcar no painel aglomerado; $\mathrm{DB}_{\mathrm{ME}}$ é a densidade básica da madeira de eucalipto e $\mathrm{ME}_{\%}$ é a porcentagem de madeira de eucalipto no painel aglomerado.

Para análise estatística dos dados o modelo experimental utilizado foi o delineamento inteiramente casualizado, com três repetições (painéis). Os dados obtidos foram analisados por meio da análise de variância $(\alpha=0,05)$. Quando houve efeito significativo da inserção do bagaço de cana-de-açúcar nos painéis aglomerados utilizou-se a análise de regressão linear simples, com exceção da razão de compactação.

\section{Resultados e Discussão}

A densidade da madeira foi superior ao encontrado para o bagaço de cana-de-açúcar (Tabela 1). Protásio et al. (2013b) também encontraram baixa densidade básica para o bagaço de cana-de-açúcar $\left(0,104 \mathrm{~g} / \mathrm{cm}^{3}\right)$. Esse resultado pode ser atribuído às distintas características anatômicas (BELINI et al., 2010) e químicas desses materiais (PROTÁSIO et al, 2013b).

Belini et al. (2010) estudaram painéis produzidos com fibras de Eucalyptus e partículas de bagaço de cana-de-açúcar. Os autores reportaram que nos painéis com componentes celulares dos colmos de cana-de-açúcar foi constatada a presença de aglomerados de parênquima e de feixes da bainha de fibras que envolvem os vasos do metaxilema. Ainda segundo
Belini et al. (2010), a menor área específica aparente dos elementos celulares das partículas de bagaço de cana-de-açúcar pode propiciar maiores espaços de vazios no interior dos painéis, formando regiões de menor densidade, e influenciar negativamente as propriedades físico-mecânicas das chapas.

A baixa densidade é um dos requisitos fundamentais para se utilizar materiais lignocelulósicos na produção de painéis aglomerados (SCATOLINO et al., 2017), pois a maior razão de compactação pode resultar na melhoria das propriedades mecânicas das chapas aglomeradas (BUFALINO et al., 2012b). Contudo, deve-se considerar, ainda, a composição química e a estrutura anatômica da matéria-prima.

O bagaço de cana-de-açúcar apresentou elevado teor de extrativos totais comparativamente a madeira de eucalipto estudada. Os extrativos são um grupo de substâncias químicas heterogêneas relacionadas aos mecanismos de defesa da planta e influenciadas por fatores genéticos e edafoclimáticos. Paula et al. (2011) e Protásio et al. (2013b) também reportaram altos teores de extrativos totais para o bagaço de cana-deaçúcar: $32 \%$ e $17 \%$, respectivamente.

Conforme mencionado por Bufalino et al. (2012a), espécies com elevados teores de extrativos podem gerar painéis cuja colagem é menos eficiente e de qualidade inferior em relação às com baixos teores. Os extrativos presentes no material lignocelulósico podem migrar para a superfície durante o processo de secagem e/ou prensagem da chapa e, consequentemente, inativar a superfície e dificultar a molhabilidade da madeira e penetração do adesivo (FRIHART; HUNT, 2010; BUFALINO et al., 2012a, 2012b).

Tabela 1. Caracterização dos materiais lignocelulósicos.

Table 1. Characterization of the lignocellulosic materials.

\begin{tabular}{lcc}
\hline & Madeira de eucalipto & Bagaço de cana-de-açúcar \\
\hline Densidade básica $\left(\mathrm{g} / \mathrm{cm}^{3}\right)$ & 0,450 & 0,120 \\
Extrativos totais $(\%)$ & 5,11 & 19,59 \\
Lignina insolúvel (\%) & 27,27 & $15,72^{\star}-20,88^{* *}$ \\
Cinzas (\%) & 0,51 & 1,89 \\
\hline
\end{tabular}

^Protásio et al. (2015) e *^Paula et al. (2011). 
Quanto ao teor de lignina, o bagaço de cana-de-açúcar apresenta desvantagem comparativamente à madeira de eucalipto. A lignina é um adesivo natural e, portanto, maiores teores são desejáveis para a produção de painéis de madeira reconstituída (KHEDARI et al., 2004; BUFALINO et al., 2012a). Espera-se que chapas produzidas com materiais lignocelulósicos com maior teor de lignina apresentem maior resistência à água e melhores propriedades mecânicas (KHEDARI et al., 2004).

O teor de cinzas do bagaço de cana-de-açúcar foi, aproximadamente, quatro vezes superior ao obtido para a madeira de eucalipto. Paula et al. (2011) e Protásio et al. (2013b) reportaram teores de cinzas para o bagaço de cana-de-açúcar de $0,8 \%$ e $1,0 \%$, respectivamente. Essas diferenças entre os valores encontrados na literatura e os obtidos neste trabalho, possivelmente, estão relacionadas ao local de plantio, bem como a posições contaminações com os minerais do solo; visto que, o bagaço de cana-de-açúcar é armazenado em pátios descobertos na usina alcooleira. A presença de maiores quantidades de minerais e alguns extrativos apolares podem resultar no bloqueio de grupos químicos reativos para a adesão com adesivos polares, afetando, assim, a qualidade da colagem e o desempenho mecânico das chapas reconstituídas (NDAZI et al., 2007).

Para densidade do painel não houve efeito significativo da inclusão de bagaço de cana-de-açúcar nos painéis aglomerados (Tabela 2). Além disso, as densidades dos painéis foram inferiores ao valor nominal $\left(0,60 \mathrm{~g} / \mathrm{cm}^{3}\right)$. Isso pode ser atribuído à especificidade das condições laboratoriais em relação ao processo industrial, com perdas de materiais durante o manuseio das partículas nas etapas de aplicação de adesivo, formação do colchão e prensagem dos painéis. Resultados similares foram reportados por Guimarães Junior et al. (2016). De acordo com a norma CS 236-66 (COMMERCIAL STANDARD - CS, 1968) todos os painéis produzidos foram classificados como de baixa densidade $\left(<0,60 \mathrm{~g} / \mathrm{cm}^{3}\right)$.

Houve acréscimo da razão de compactação com o aumento da inserção de bagaço de cana-de-açúcar no painel. Esse resultado foi mencionado por outros autores que estudaram o uso de resíduos lignocelulósicos na produção de painéis aglomerados (SILVA et al., 2015, 2016; GUIMARÃES JUNIOR et al., 2016; SCATOLINO et al., 2013; SCATOLINO et al., 2017). Esses pesquisadores constataram que o referido comportamento pode ser explicado devido à baixa densidade do resíduo comparativamente a madeira (Tabela 1). Apenas o tratamento com inserção de $20 \%$ de bagaço de cana-de-açúcar atendeu a faixa ideal de razão de compactação $(1,3$ a 1,6), preconizada por Maloney (1993).

Para a absorção de água (Figura 1) não houve efeito significativo da adição de bagaço de cana-de-açúcar nos painéis aglomerados. Os valores médios foram de 111,5\% e 132,8\% para absorção após 2 e 24 horas, respectivamente. Scatolino et al. (2017) relataram valores de absorção de água, após 2 e 24 horas de imersão em água, de 120,5\% e 138,1\%, respectiva-

Tabela 2. Densidade aparente e razão de compactação para os painéis aglomerados.

Table 2. Apparent density and compaction ratio for the particleboards.

\begin{tabular}{ccc}
\hline Bagaço de cana-de-açúcar & $\begin{array}{c}\text { Densidade aparente } \\
\left(\mathrm{g} / \mathrm{cm}^{3}\right)\end{array}$ & $\begin{array}{c}\text { Razão de } \\
\text { compactação }\end{array}$ \\
\hline $0 \%$ & $0,550(0,03)$ & $1,25(0,07) \mathrm{A}$ \\
$20 \%$ & $0,530(0,03)$ & $1,38(0,01) \mathrm{B}$ \\
$40 \%$ & $0,520(0,04)$ & $1,64(0,08) \mathrm{C}$ \\
$60 \%$ & $0,530(0,01)$ & $2,44(0,05) \mathrm{D}$ \\
\hline
\end{tabular}

Médias seguidas da mesma letra não diferem entre si pelo teste Scott-knott a 5\% de significância. Valores entre parênteses representam o desvio padrão. 
mente, para painéis de baixa densidade produzidos com a madeira de eucalipto, ou seja, próximos aos observados neste trabalho.

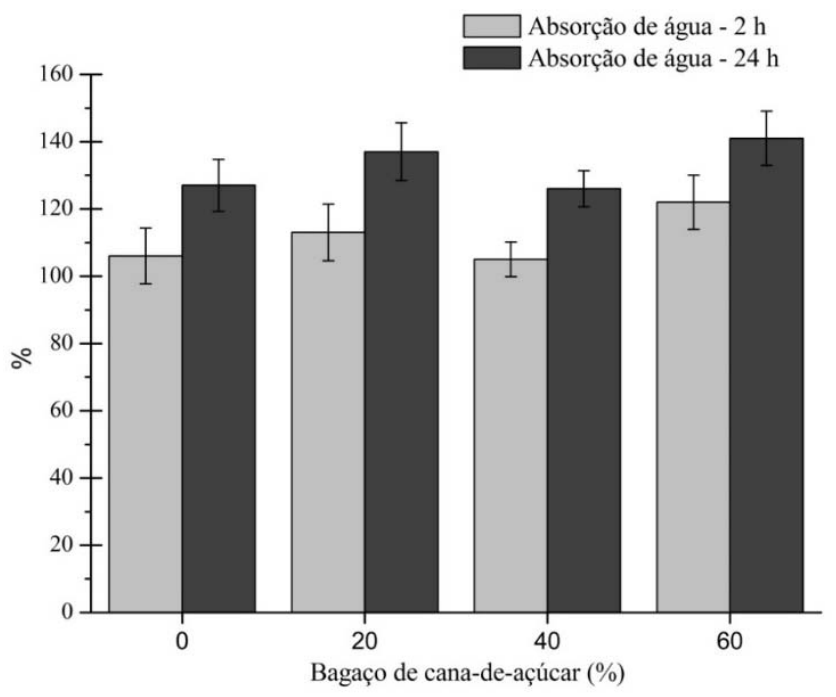

Figura 1. Absorção de água após 2 e 24 horas de imersão em água.

Figure 1. Water absorption after 2 and 24 hours of immersion.

Diferentemente do obtido neste trabalho, Mendes et al. (2012) e Guimarães Junior et al. (2016) observaram, para painéis aglomerados, acréscimo da absorção de água com a substituição parcial de madeira por bagaço de cana-de-açúcar e bagaço de sorgo, respectivamente. Os autores explicaram que elevados valores de razão de compactação, decorrentes da inserção de resíduos de baixa densidade nas chapas, resultam na necessidade de um número maior de partículas para que se obtenha a densidade do painel, ocasionando assim a disponibilidade de mais sítios hidroxílicos. Contudo, deve-se considerar, ainda, a composição química dos materiais lignocelulósicos e a porcentagem de substituição da madeira pelo resíduo.

Para o inchamento em espessura, após 2 e 24 horas de imersão em água, o acréscimo de bagaço de cana-de-açúcar nos painéis aglomerados provocou aumento nessa propriedade (Figura 2). Analisando-se o coeficiente $\beta_{1}$ dos modelos de regressão ajustados, percebe-se que o aumento na inserção de bagaço de cana-de-açúcar no painel na ordem de $1 \%$, proporciona elevação de 0,19 e $0,29 \%$ de inchamento em espessura após 2 e 24 horas de imersão, respectivamente. Esse comportamento pode ter ocorrido em função do acréscimo da razão de compactação com o aumento da proporção de resíduo no painel.

Protásio et al. (2012) encontraram correlação positiva entre o inchamento em espessura das chapas aglomeradas produzidas com madeira de Eucalyptus com o acréscimo da razão de compactação. A maior razão de compactação resulta em maior inchamento higroscópico da parede celular, devido ao maior número de sítios de sorção, e a maior liberação das tensões de compressão geradas durante o processo de prensagem (KELLY, 1977; MELO, 2013).

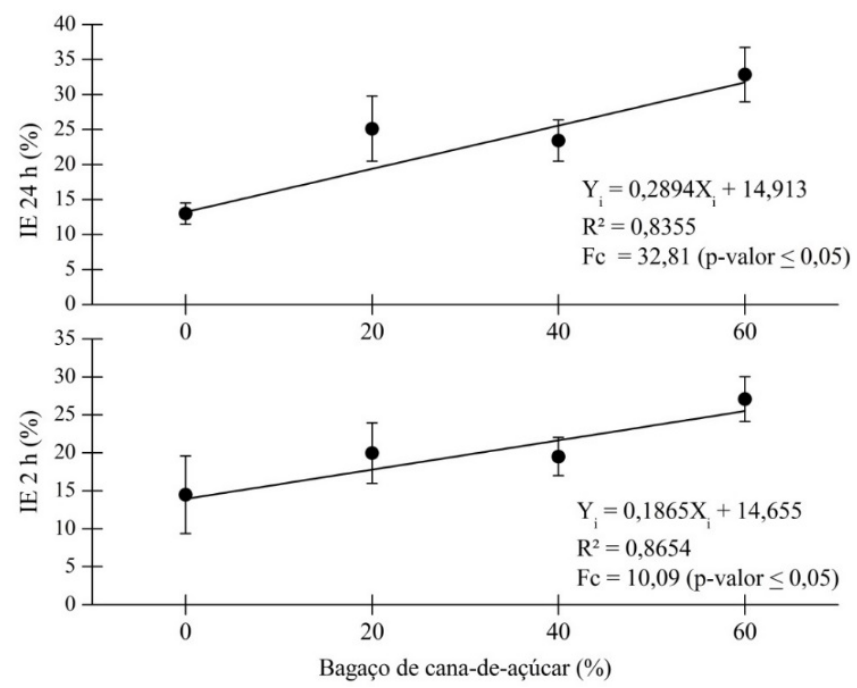

Figura 2. Inchamento em espessura após 2 horas (IE 2 h) e 24 horas (IE 24 h) de imersão em água.

Figure 2. Thickness swelling after 2 hours (TS $2 \mathrm{~h}$ ) and 24 hours (TS $24 \mathrm{~h}$ ) of immersion in water.

A norma Commercial Standard CS 236-66 (CS, 1968) estipula, para painéis aglomerados de baixa densidade e produzidos com adesivo ureia-formaldeído, valor máximo de 30\% para o inchamento em espessura após 24 horas de imersão em água. Neste sentido, igualando-se este valor na equação obtida, nota-se que a inserção máxima de bagaço no painel para atendimento das exigências normativas é de 52,1\%. 
Para as propriedades mecânicas do ensaio de flexão estática (MOE e MOR) houve efeito significativo da substituição de bagaço de cana-de-açúcar nos painéis aglomerados de baixa densidade (Figura 3). O acréscimo de $1 \%$ de resíduo no painel, promove uma redução de, aproximadamente, $8,5 \mathrm{MPa}$ para o MOE e de 0,1 MPa para o MOR.
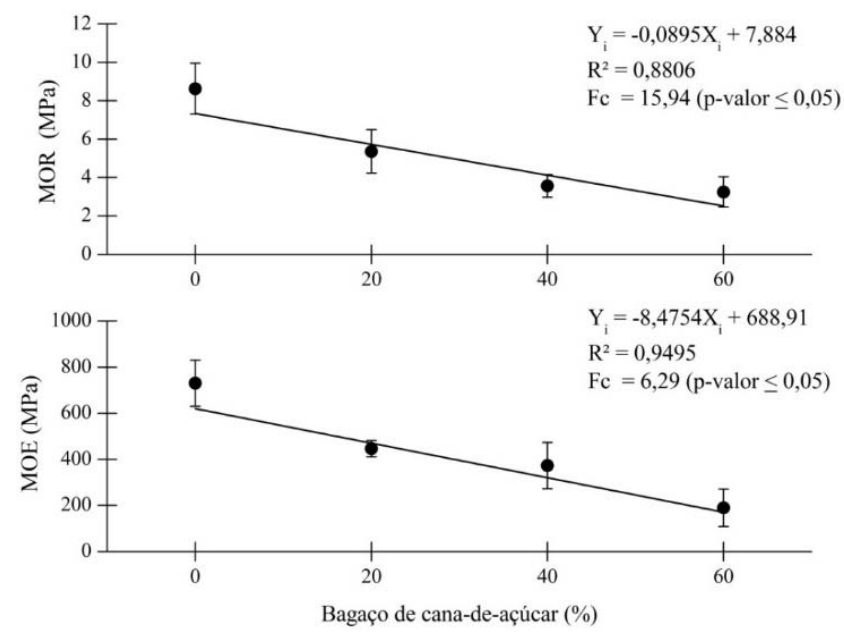

Figura 3. Módulos de elasticidade (MOE) e de ruptura (MOR) na flexão estática.

Figure 3. Modulus of elasticity (MOE) and rupture (MOR) at static bending.

O decréscimo do MOE e do MOR em função da adição de resíduos lignocelulósicos nos painéis aglomerados tem sido relatado na literatura especializada (SCATOLINO et al., 2013, GUIMARÃES JUNIOR et al., 2016; SILVA et al., 2016; SCATOLINO et al., 2017; CÉSAR et al., 2017). Segundo Guimarães Junior et al. (2016) e Scatolino et al. (2017) uma possível explicação para a diminuição das propriedades mecânicas dos painéis aglomerados convencionais consiste na baixa densidade do resíduo e, consequentemente, aumento da razão de compactação, resultando na menor disponibilidade de adesivo por partícula.

César et al. (2017) relataram que a maior quantidade de adesivo possibilita melhor adesão entre as partículas e, consequentemente, maiores valores de MOE, MOR e tração perpendicular. Adicionalmente, as distintas características anatômicas da matéria-prima lignocelulósica podem auxiliar na explicação dos resultados.
De acordo com a norma de comercialização CS 236-66 (CS, 1968), os painéis aglomerados de baixa densidade e produzidos com o adesivo ureia-formaldeído devem apresentar valor mínimo para o módulo de elasticidade de 1029,7 MPa. Dessa forma, constata-se que nenhum painel produzido atendeu a referida normativa. Esse resultado pode ser atribuído às peculiaridades laboratoriais associadas à produção dos painéis. Contudo, o painel produzido apenas com madeira atingiu o valor mínimo exigido pela norma A208 (ANSI, 1999) para o MOE, que é de $550 \mathrm{MPa}$.

Já para o módulo de ruptura quando se iguala ao valor mínimo exigido pela norma CS 236-66 (CS, 1968) de 5,5 MPa na equação ajustada, observa-se que o máximo de bagaço de cana-de-açúcar que se recomenda na produção dos painéis aglomerados de baixa densidade é de $26,6 \%$.

Scatolino et al. (2017), avaliando painéis aglomerados de baixa densidade produzidos com madeira de eucalipto e pergaminho dos grãos de café, observaram valores para o módulo de elasticidade no ensaio de flexão estática variando de $650 \mathrm{MPa}$ a $401 \mathrm{MPa}$ para chapas com 0\% e 50\% de resíduo, respectivamente. Para o módulo de ruptura, os autores obtiveram valores entre 8,2 $\mathrm{MPa}$ e 4,4 MPa para os painéis produzidos apenas com madeira e com $50 \%$ de pergaminho dos grãos de café, respectivamente. As tendências observadas por esses autores, bem como os valores encontrados para essas propriedades mecânicas, assemelham-se ao presente estudo.

A tração perpendicular foi afetada significativamente com o aumento proporção do resíduo em relação à madeira de eucalipto nos painéis aglomerados de baixa densidade (Figura 4). O acréscimo de $1 \%$ de bagaço de cana-de-açúcar resulta em uma redução desta propriedade na ordem de 0,0013 MPa. Guimarães Junior et al. (2016) observaram valor médio de tração perpendicular de 0,29 MPa para painéis produzidos com madeira de Eucalyptus, ou seja, superior ao obtido neste 
trabalho. Vale salientar que os autores utilizaram $12 \%$ de adesivo ureia-formaldeído na produção das chapas aglomeradas de baixa densidade.

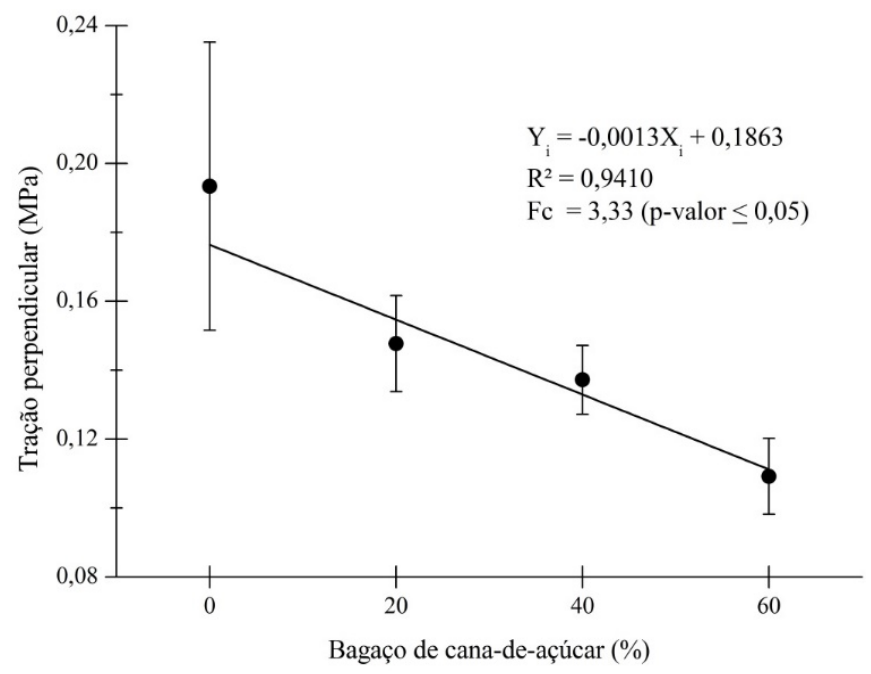

Figura 4. Tração perpendicular dos painéis aglomerados de baixa densidade.

Figure 4. Internal bond of the low density particleboards.

Possivelmente, o decréscimo da tração perpendicular ocorreu devido aos elevados teores de extrativos e cinzas e baixo teor de lignina do bagaço de cana-de-açúcar. Marra (1992) afirmou que materiais lignocelulósicos com elevados teores de extrativos apresentam dificuldades de colagem, resultando em baixa resistência da ligação adesiva entre as partículas. De forma semelhante, César et al. (2017) observaram redução da propriedade de tração perpendicular com o acréscimo de casca de arroz em painéis aglomerados de média densidade. Os autores atribuíram os resultados aos elevados teores de minerais e extrativos e ao baixo teor de lignina da casca de arroz.

Além disso, a presença de aglomerados de parênquima e de feixes da bainha de fibras que envolvem os vasos do metaxilema, no bagaço de cana-de-açúcar, pode resultar em maiores espaços de vazios no interior dos painéis (BELINI et al., 2010) e, consequentemente, diminuir a resistência à tração perpendicular.
A norma de comercialização CS 236-66 estabelece valor mínimo de 0,14 MPa para tração perpendicular nos painéis de baixa densidade e produzidos com adesivo ureia-formaldeído. Neste sentido, observa-se que os painéis produzidos com até $40 \%$ de bagaço de cana-de-açúcar atenderam a norma supracitada.

\section{Conclusões}

Há decréscimo da estabilidade dimensional dos painéis aglomerados com o aumento da proporção de bagaço de cana-de-açúcar para chapas de baixa densidade.

As propriedades mecânicas são afetadas negativamente com a inserção do bagaço de cana-de-açúcar em detrimento da madeira de Eucalyptus.

Recomenda-se o máximo de $26 \%$ de bagaço de cana-deaçúcar, em associação com a madeira de Eucalyptus, na produção de painéis aglomerados de baixa densidade.

\section{Agradecimentos}

Os autores expressam seus sinceros agradecimentos à FAPEG, ao CNPq e a CAPES pelo financiamento do projeto e/ou concessão de bolsas de estudos aos autores. Esses recursos foram fundamentais para o desenvolvimento da pesquisa.

\section{Referências}

AMERICAN NATIONAL STANDARD PARTICLEBOARD - ANSI. A208.1 Particleboard. 1999. 11p.

AMERICAN SOCIETY FOR TESTING AND MATERIALS - ASTM. ASTM D 1037: standard methods of evaluating properties of wood-base fiber and particles materials. Philadelphia: 2006.

ASSOCIAÇÃO BRASILEIRA DE NORMAS TÉCNICAS ABNT. NBR 11941: Madeira - Determinação da densidade básica. Rio de Janeiro: 2003. 6p. 
ASSOCIAÇÃO BRASILEIRA DE NORMAS TÉCNICAS NBR 13999: papel, cartão, pastas celulósicas e madeira: determinação do resíduo (cinza) após a incineração a $525^{\circ} \mathrm{C}$. Rio de Janeiro, 2003. $4 \mathrm{p}$.

ASSOCIAÇÃO BRASILEIRA DE NORMAS TÉCNICAS. NBR 14853: Madeira - determinação do material solúvel em etanol-tolueno e em diclorometano e em acetona. Rio de Janeiro, 2010a. 3 p.

ASSOCIAÇÃO BRASILEIRA DE NORMAS TÉCNICAS. NBR 7989: pasta celulósica e madeira: determinação de lignina insolúvel em ácido. Rio de Janeiro, 2010b. 6 p.

BATTISTELLE, R. A. G.; MARCILIO, C.; LAHR, F. A. R. Emprego do bagaço da cana-de-açúcar (Saccharum officinarum) e das folhas caulinares do bambu da espécie Dendrocalamus giganteus na produção de chapas de partículas. Revista Minerva, v. 5, n. 3, p. 297-305, 2009.

BELINI, U.; TOMAZELLO FILHO, M.; LOUZADA, J. L. P. C.; RODRIGUES, J. C. Aspectos anatômicos e tecnológicos de painéis confeccionados com fibras de eucalipto e cana-deaçúcar. Cerne, v.16, Suplemento, p. 48-52, 2010.

BUFALINO, L.; ALBINO, V. C. S.; SÁ, V. A.; CORRÊA, A. R. R.; MENDES, L. M.; ALMEIDA, N. A. Particleboards made from Australian red cedar: processing variables and evaluation of mixed species. Journal of Tropical Forest Science, v. 24, n. 2, p. 162-172, 2012b.

BUFALINO, L.; PROTÁSIO, T. P. P.; COUTO, A. M.; NASSUR, O. A. C.; SÁ, V. A.; TRUGILHO, P. F.; MENDES, L. M. Caracterização química e energética para aproveitamento da madeira de costaneira e desbaste de cedro australiano. Pesquisa Florestal Brasileira, v. 32, n. 70, p. 129-137, 2012a. doi: 10.4336/2012.pfb.32.70.13

CARVALHO, A. G.; ANDRADE, B. G.; CABRAL, C. P. T.; VITAL, B. R. Efeito da adição de resíduos de poda da ervamate em painéis aglomerados. Revista Árvore, v.39, n.1, p. 209-214, 2015. doi: 10.1590/0100-67622015000100020

CÉSAR, A. A. S.; BUFALINO, L.; MACEDO, L. B.; MESQUITA, R. G. A.; PROTÁSIO, T. P.; MENDES, L. M. Use of coffee plant stem in the production of conventional particleboards. Key Engineering Materials, v. 600, p. 703-708, 2014. doi:10.4028/www.scientific.net/KEM.600.703

CÉSAR, A. A. S.; BUFALINO, L.; MENDES, L. M.; MESQUITA, R. G. A.; PROTÁSIO, T. P.; MENDES, R. F.; ANDRADE, L. M. F. Transforming rice husk into a high-added value product: potential for particleboard production. Ciência Florestal, v. 27, n. 1, p. 303-313, 2017. doi: $10.5902 / 1980509826468$

COMMERCIAL STANDARD - CS. CS 236-66: mat formed wood particleboard. [S.l.], 1968.

COMPANHIA NACIONAL DE ABASTECIMENTO. Acompanhamento da safra brasileira de cana-de-açúcar. Conab: Brasília, v. 3, n. 4, p. 1-76, 2017.

DEUTSCHES INSTITUT FUR NORMUNG - DIN. DIN 52362: Testing of wood chipboards, bending test, determination of bending strength. Berlin, 1982. $40 \mathrm{p}$.

DIAS, J. M. C. S.; SOUZA, D. T.; BRAGA, M.; ONOYOMA, M. M.; MIRANDA, C. H. B.; BARBOSA, P. F. D.; ROCHA, J. D. Produção de briquetes e pelletes a partir de resíduos agrícolas, agroindustriais e florestais. Brasília: Embrapa Agroenergia, 2012. $130 \mathrm{p}$.

FIORELLI, J.; LAHAR, F. A.; NASCIMENTO, M. F.; SAVASTANO JUNIOR, H.; ROSSIGNOLO, J. A. Painéis de partículas à base de bagaço de cana e resina de mamona-produção e propriedades. Acta Scientiarum, v. 33, n. 4, p. 401-406, 2011. doi: 10.4025/actascitechnol.v33i4.9615

FREIRE, C. S.; SILVA, D. W.; SCATOLINO, M. V.; CÉSAR, A. A. S.; BUFALINO, L.; MENDES, L. M. Propriedades físicas de painéis aglomerados comerciais confeccionados com bagaço de cana e madeira. Floresta e Ambiente, v. 18, n. 2, p. 178-185, 2011. doi 10.4322/floram.2011.036

FRIHART, C. R.; HUNT, C. G. Adhesives with wood materials: bond formation and performance. In: FOREST PRODUCTS LABORATORY. Wood handbook: wood as an engineering material. Washington, DC: USDA, 2010. p. 10/1$10 / 24$.

GUIMARÃES JUNIOR, J. B.; XAVIER, M. M.; SANTOS, T. S.; PROTÁSIO, T. P.; MENDES, R. F.; MENDES, L. M. Inclusão de resíduo da cultura de sorgo em painéis aglomerados de eucalipto. Pesquisa Florestal Brasileira, v. 36, n. 88, p. 435442, 2016. doi: 10.4336/2016.pfb.36.88.1036

GUIMARÃES, B. M. R.; MENDES, L. M.; TONOLI, G. H. D.; BUFALINO, L.; MENDES, R. F.; GUIMARÃES JUNIOR, J. B. Chemical treatment of banana tree pseudostem particles aiming the production of particleboards. Ciência e Agrotecnologia, v. 38 , n.1, p. 43-49, 2014. doi: 10.1590/S141370542014000100005 
KELLY, M. W. Critical literature review of relationships between processing parameters and physical properties of particleboard. USDA Forest Service General Technology Report Forest Products Laboratory, Madison, USA, 1977. 66 p.

KHEDARI, J.; NANKONGNAB, N.; HIRUNLABH, J.; TEEKASAP, S. New low-cost insulation particleboards from mixture of durian peel and coconut coir. Building and Environment, v. 39, n. 1, p. 59-65, 2004. doi: 10.1016/j.buildenv.2003.08.001

MALONEY, T. M. Modern particleboard and dry-process fiberboard manufacturing. 2. Ed. São Francisco: Miller Freeman, 1993.689 p.

MARRA, A. A. Technology of wood bonding. New York: Van Nostrand Reinhold, 1992. 453 p.

MELO, R. R. Estabilidade dimensional de compostos de madeira. Ciência da Madeira, v. 4, n. 2, p. 152-175, 2013.

MENDES, R. F.; MENDES, L. M.; GUIMARÃES JR., J. B.; SANTOS, R. C.; CÉSAR, A. A. S. Efeito da associação de bagaço de cana, tipo e teor de adesivo na produção de painéis aglomerados com madeira de pinus. Ciência Florestal, v. 22, n. 1, p. 187-196, 2012. doi: 10.5902/198050985088

NDAZI, B. S.; KARLSSON, S.; TESHA, J. V.; NYAHUMWA, C. W. Chemical and physical modifications of rice husks for use as composite panels. Composites Part A: Applied Science and Manufacturing, v.38, n. 33, p.925-935, 2007.

PAULA, L. E. R.; TRUGILHO, P. F.; NAPOLI, A.; BIANCHI, M. L. Characterization of residues from plant biomass for use in energy generation. Cerne, v. 17, n. 2, p. 237-246, 2011. doi: 10.1590/S0104-77602011000200012

PROTÁSIO, T. P.; BUFALINO, L.; GUIMARÃES JUNIOR, M.; TONOLI, G. H. D.; TRUGILHO, P. F. Técnicas multivariadas aplicadas à avaliação de resíduos lignocelulósicos para a produção de bioenergia. Ciência Florestal, v. 23, n. 4, p. 771-781, 2013a. doi: 10.5902/1980509812361

PROTÁSIO, T. P.; BUFALINO, L.; TONOLI, G. H. D.; GUIMARÃES JÚNIOR, M.; TRUGILHO, P. F.; MENDES, L. M. Brazilian lignocellulosic wastes for bioenergy production: characterization and comparison with fossil fuels. Bioresources, v. 8, n.1, p. 1166-1185, 2013 b.

PROTÁSIO, T. P.; GUIMARÃES JUNIOR, J. B.; MENDES, R. F.; MENDES, L. M.; GUIMARÃES, B. M. R. Correlações entre as propriedades físicas e mecânicas de painéis aglomerados de diferentes espécies de Eucalyptus. Floresta e Ambiente, v. 19 , n. 2, p. 123-132, 2012. doi: 10.4322/floram.2012.014

PROTÁSIO, T. P.; MENDES, R. F.; SCATOLINO, M. V.; MENDES, L. M.; TRUGILHO, P. F.; MELO, I. C. N. A. Estabilidade térmica de painéis aglomerados de bagaço de canade-açúcar e madeira de Pinus spp. Scientia Forestalis, v. 43, n. 107, p.683-691, 2015.

RIBEIRO, D.V.; MORELLI, M.R. Resíduos sólidos: problema ou oportunidade?. Rio de Janeiro: Interciência, 2009. $158 \mathrm{p}$.

SCATOLINO, M. V.; COSTA, A. O.; GUIMARÃES JUNIOR, J. B.; PROTÁSIO, T. P.; MENDES, R. F.; MENDES, L. M. Eucalyptus wood and coffee parchment for particleboard production: physical and mechanical properties. Ciência e Agrotecnologia, v. 41, n. 2, p. 139-146, 2017. doi: 10.1590/141370542017412038616

SCATOLINO, M. V.; PROTÁSIO, T. P.; MENDES, R. F.; MENDES, L. M. Thermal stability of Pinus oocarpa and maize cob particleboards. Ciência e Agrotecnologia, v. 39, n.4, p. 348-354, 2015. doi: 10.1590/S1413-70542015000400005

SCATOLINO, M.V.; SILVA, D.W.; MENDES, R. F.; MENDES, L. M. Use of maize cob for production of particleboard. Ciência e Agrotecnologia, v.37, n.4, p. 330-337, 2013. doi: 10.1590/S1413-70542013000400006

SILVA, D. W.; FARRAPO, C. L.; PEREIRA, D.; MENDES, R. F.; MENDES, L. M. MDP com partículas de eucalipto e palha de milho. Scientia Forestalis, v. 43, n. 108, p. 853-862, 2015. doi: $10.18671 /$ scifor.v43n108.10

SILVA, D. W.; SCATOLINO, M. V.; PRADO, N. R. T.; MENDES, R. F.; MENDES, L. M. Addition of different proportions of castor husk and pine wood in particleboards. Waste Biomass Valorization, v. 16, p. 1-7, 2016. doi:10.1007/s12649-016-9742-7 